\title{
Modelos de regressão aleatória na avaliação genética do crescimento de ovinos da raça Santa Inês
}

\author{
José Lindenberg Rocha Sarmento ${ }^{1,6}$, Robledo de Almeida Torres ${ }^{2,6}$, Raimundo Nonato \\ Braga Lôbo ${ }^{3,6}$, Lucia Galvão de Albuquerque ${ }^{4,6}$, Wandrick Hauus de Sousa ${ }^{5,6}$, José \\ Ernandes Rufino de Sousa ${ }^{1}$
}

\footnotetext{
${ }^{1}$ UFPI, Campus de Bom Jesus, CEP: 64.900-000, Bom Jesus, PI.

2 Departamento de Zootecnia/UFV, CEP: 36571-000, Viçosa, MG.

3 EMBRAPA Caprinos.

${ }^{4}$ FCAVIUNESP, CEP: 14884-900, Jaboticabal, SP.

${ }^{5}$ EMEPA-PB.

${ }^{6}$ Bolsista do CNPq
}

RESUMO - Utilizaram-se 17.767 registros de peso de 4.210 cordeiros da raça Santa Inês com o objetivo de comparar modelos de regressão aleatória com diferentes estruturas para modelar a variância residual em estudos genéticos da curva de crescimento. Os efeitos fixos incluídos na análise foram: grupo contemporâneo e idade da ovelha no parto. As regressões fixas e aleatórias foram ajustadas por meio de polinômios de Legendre de ordens 4 e 3, respectivamente. A variância residual foi ajustada por meio de classes heterogêneas e por funções de variância empregando polinômios ordinários e de Legendre de ordens 2 a 8 . O modelo considerando homogeneidade de variâncias residuais mostrou-se inadequado. De acordo com os critérios utilizados, a variância residual contendo sete classes heterogêneas proporcionou melhor ajuste, embora um mais parcimonioso, com cinco classes, pudesse ser utilizado sem perdas na qualidade de ajuste da variância nos dados. O ajuste de funções de variância com qualquer ordem foi melhor que o obtido por meio de classes. O polinômio ordinário de ordem 6 proporcionou melhor ajuste entre as estruturas testadas. A modelagem do resíduo interferiu nas estimativas de variâncias e parâmetros genéticos. Além da alteração da classificação dos reprodutores, a magnitude dos valores genéticos preditos apresenta variações significativas, de acordo com o ajuste da variância residual empregado.

Palavras-chave: herdabilidade, heterogeneidade de variâncias, modelo animal, ovinos deslanadas, parâmetros genéticos, valor genético

\section{Random regression models on genetic evaluation on the growth of Santa Inês breed sheep}

\begin{abstract}
It was used 17,767 weight records of 4,210 Santa Inês breed lambs aiming to compare random regression models with different structures to model the residual variance in genetic studies of the growth curve. The fixed effects included in the analysis were contemporary group and age of the ewe at lambing. Fixed and random regressions were fitted through Legendry polynomials of orders 4 and 3, respectively. The residual variance was fitted by heterogeneous classes and by functions of variances employing ordinary polynomials and Legendry polynomials of the orders 2 to 8 . The model considering homogeneity of residual variances was inadequate. Accordingly to the used criteria, the residual variance containing seven heterogeneous classes provided the best fit, although a more parsimonious one, with five classes, could be used without losses on the quality of variance fit on the data. The fit of functions of variances with any order was better than that obtained through classes. The ordinary polynomial of order 6 provided the best fit among the tested structures. The modeling of the residue interfered on the estimative of the variances and genetic parameters. In addition to changes in the classification of the reproducers, the magnitude of the predicted genetic values shows significant variations, accordingly to the fitting of the used residual variance.
\end{abstract}

Key Words: animal model, breeding value, genetic parameters, hair sheep, heritability, heterogeneity of variance

\section{Introdução}

Os modelos de regressão aleatória têm sido os mais indicados para análise de características longitudinais no melhoramento animal. Esses modelos permitem ajustar curvas de crescimento aleatórias para cada indivíduo e obter estruturas de variâncias e covariâncias entre as diferentes medidas por meio de funções de covariâncias para os efeitos 
aleatórios de interesse. Além disso, o emprego desses modelos permite a modelagem da variância residual sob diferentes estruturas.

Vários trabalhos têm mostrado a necessidade de considerar heterogeneidade de variâncias residuais em modelos de regressão aleatória (Meyer, 2000, em bovinos de corte; Lewis \& Brotherstone, 2002, em ovinos de corte; e El Faro \& Albuquerque, 2003, em bovinos de leite). Para características de crescimento, estas variâncias em bovinos e ovinos de corte tendem a aumentar com a idade, como verificado por Meyer (2001) e Fischer et al. (2004). Dessa forma, a modelagem da curva de crescimento, considerando a variância residual heterogênea em função da idade, pode melhorar a partição da variância fenotípica nas variâncias devido aos efeitos aleatórios incluídos no modelo de análise. Por outro lado, o emprego de variâncias residuais heterogêneas implica o aumento do número de parâmetros a serem estimados no processo de maximização da função de verossimilhança, o que pode dificultar a utilização dos modelos de regressão aleatória em avaliações genéticas.

Assim, busca-se a utilização de modelos mais parcimoniosos, sem perda da qualidade de ajuste dos efeitos aleatórios considerados. Dessa forma, em diversos estudos, têm-se proposto a modelagem das variâncias residuais, utilizando-se classes heterogêneas ou por meio de funções de variância residuais (Meyer, 1999, 2001; Albuquerque \& Meyer, 2001). Dentre as funções que podem ser empregadas para ajustar uma função de variância, destacam-se os polinômios ordinários e os polinômios ortogonais de Legendre (Kirkpatrick et al., 1990), os quais não requerem pressuposição sobre a forma da curva a ser ajustada.

Desta forma, objetivou-se com este trabalho comparar estruturas para modelar a variância residual em modelos de regressão aleatória e avaliar a influência desta modelagem na estimação de parâmetros genéticos e predição de valores genéticos para a curva de crescimento de ovinos Santa Inês.

\section{Material e Métodos}

Os dados utilizados neste estudo são provenientes de três rebanhos experimentais de ovinos da raça Santa Inês, pertencentes à Empresa Estadual de Pesquisa Agropecuária da Paraíba (EMEPA-PB) e à Empresa de Pesquisa Agropecuária Brasileira(EMBRAPA Caprinos e EMBRAPA Tabuleiros Costeiros), relativos ao período de 1983 a 2005.

$\mathrm{O}$ arquivo inicial constava de 23.198 registros de pesos em diferentes idades, mensurados, em média, a cada 28 dias de idade. Foram consideradas para análise informações de cordeiros pesados até 196 dias de idade; nascidos de parto simples e duplo; peso médio diário dentro da média de todos os animais de mesmo grupo de contemporâneo \pm três desvios padrão; pertencentes a grupos de contemporâneos com, no mínimo, cinco animais.

A definição de grupo contemporâneo incluiu: rebanho, ano e estação da pesagem, sexo do cordeiro, tipo de nascimento (simples e duplo) e classes de idade (sugerido por Meyer, 2005, para diminuir a amplitude de idades dos animais comparados diretamente dentro de cada grupo, o que refletiu diretamente na elevação, exacerbada, da variância fenotípica). Após a edição do arquivo de dados, restaram para análise 17.767 pesos em diferentes idades de 4.210 cordeiros filhos, 130 reprodutores e 1.552 matrizes.

A regressão fixa e a aleatória foram representadas por funções contínuas, cujas idades foram descritas em termos de polinômios ortogonais de Legendre. Generalizando, os modelos com diferentes estruturas de variâncias residuais podem ser representados como se segue:

$y_{i j}=F+\sum_{m=1}^{4} \beta_{m} \phi_{m}+\sum_{m=1}^{3} \alpha_{i m} \phi_{m}+\sum_{m=1}^{3} \rho_{i m} \phi_{m}+\sum_{m=1}^{3} \gamma_{i m} \phi_{m}+\sum_{m=1}^{3} \delta_{i m} \phi_{m}+\varepsilon_{i j}$ em que $y_{i j}=$ peso no dia $\mathrm{j}$ do cordeiro $\mathrm{i} ; \mathrm{F}=$ conjunto de efeitos fixos, constituído pelo grupo de contemporâneo (1.112 subclasses) e pela covariável idade da ovelha ao parto, efeitos linear e quadrático; $\beta_{m}=\mathrm{m}$ coeficiente de regressão fixo do peso sobre o polinômio de Legendre (função cúbica, como verificado por Sarmento et al., 2010) para modelar a curva média de crescimento da população; $\alpha_{i m}, \rho_{i m}, \gamma_{i m}$ e $\delta_{i m}=m$ coeficientes de regressão genético aditivo direto, ambiente permanente de animal, genético aditivo materno e ambiente permanente materno, respectivamente, para o cordeiro i; $\phi_{m}=\mathrm{m}$ função polinomial de Legendre da idade padronizada $(-1<$ idade $<1)$; e $\varepsilon_{i j}=$ efeito aleatório residual.

Avaliou-se a modelagem da variância residual, considerando-a homogênea e heterogênea por meio de diferentes estruturas. Inicialmente, considerou-se heterogeneidade entre classes de idade $\left(\mathrm{CL}_{\mathrm{m}}\right.$, sendo $\mathrm{m} \mathrm{o}$ número de classes), agrupadas como se segue: CL1, homogênea; CL2, 1-112 e 113-196 dias; CL3, 1-56, 57-112 e 113-196 dias; CL4, 1-28, 29-56, 57-112 e 113-196 dias; CL5, 1-28, 29-56, 57-84, 85-112 e113-196 dias; CL6, 1-28, 29-56, 5784, 85-112, 113-140 e 141-196 dias; e CL7, 1-28, 29-56, 57-84, 85-112, 113-140, 141-168 e 169-196 dias de idade.

Posteriormente, as variâncias residuais foram estimadas por meio de coeficientes de regressão de funções de variâncias, ajustadas por polinômios ordinários (FO) e de Legendre (FL), com ordens variando de dois a seis, ou seja, linear a quíntupla $\left(\mathrm{FO}_{\mathrm{m}}\right.$ ou $\mathrm{FL}_{\mathrm{m}}$, sendo $\mathrm{m}$ a ordem de ajuste), que podem ser representadas por: $\sigma_{j}^{2}=\sigma_{0}^{2}\left(1+\sum_{r=1}^{m} \beta_{r}\left(a_{i j}^{*}\right)^{r}\right)$, em que $\sigma_{j}^{2}=$ variância residual na idade j; $\sigma_{0}^{2} \stackrel{r=1}{=}$ variância do

R. Bras. Zootec., v.39, n.8, p.1723-1732, 2010 
intercepto; $\beta_{r}=$ m coeficientes de regressão da função de variância; e $a_{i j}^{*}=$ idades de medições. No total, foram avaliados 17 diferentes modelos empregando classes e funções de variâncias.

$\mathrm{Na}$ forma matricial, o modelo anterior, com suas respectivas pressuposições, pode ser escrito como:

$$
\begin{gathered}
y=X b+Z_{1} a+Z_{2} c+Z_{3} m+Z_{4} q+e \\
E\left[\begin{array}{c}
y \\
a \\
c \\
m \\
q \\
e
\end{array}\right]=\left[\begin{array}{c}
X b \\
0 \\
0 \\
0 \\
0 \\
0
\end{array}\right] \quad e \quad \begin{array}{l}
\operatorname{Var}(a)=K_{a} \otimes A, \\
\operatorname{Var}(m)=K_{c} \otimes I_{N d} \otimes A,
\end{array} \\
\quad \begin{array}{l}
\operatorname{Var}(q)=K_{q} \otimes I_{N m}, \\
\operatorname{Var}(e)=R ;
\end{array}
\end{gathered}
$$

em que $y=$ vetor de $\mathrm{N}$ observações referentes a $\mathrm{N}_{\mathrm{d}}$ animais; $b=$ vetor que contém os efeitos fixos e os coeficientes $b_{m}$ da regressão fixa; $a$ = vetor $\mathrm{k}_{\mathrm{a}} \times \mathrm{N}_{\mathrm{D}}$ de coeficientes de regressão aleatória genéticos aditivos diretos, em que $\mathrm{N}_{\mathrm{D}}$ denota o número total de animais na matriz de parentesco (5.357); $c=$ vetor $\mathrm{k}_{\mathrm{C}} \times \mathrm{N}_{\mathrm{d}}$ de coeficientes de regressão aleatória ambiente permanente de animal; $m=$ vetor $\mathrm{k}_{\mathrm{m}} \times \mathrm{N}_{\mathrm{D}}$ de coeficientes de regressão aleatória genéticos aditivos maternos; $q$ = vetor $\mathrm{k}_{\mathrm{q}} \times \mathrm{N}_{\mathrm{m}}$ de coeficientes de regressão aleatória de ambiente permanente materno, sendo $\mathrm{N}_{\mathrm{m}}$ igual ao número de mães com progênies que têm observações controladas; e = vetor de erros aleatórios; $\mathrm{X}, \mathrm{Z}_{1}, \mathrm{Z}_{2}, \mathrm{Z}_{3}$ e $\mathrm{Z}_{4}=$ matrizes de incidência dos coeficientes de regressão fixos, coeficientes de regressão aleatória genético aditivo direto, ambiente permanente de animal, genético aditivo materno e ambiente permanente materno, respectivamente. $\mathrm{K}_{\mathrm{a}}$, $\mathrm{K}_{\mathrm{c}}$, $\mathrm{K}_{\mathrm{m}}$ e $\mathrm{K}_{\mathrm{q}}=$ matrizes de covariâncias entre os coeficientes de regressão aleatórios genético aditivo direto, ambiente permanente de animal, genético aditivo materno e ambiente permanente materno, respectivamente; $\mathrm{A}=$ matriz dos numeradores do coeficiente de parentesco entre os indivíduos; $\mathrm{I}_{\mathrm{Nd}}$ é uma matriz identidade de dimensão $\mathrm{N}_{\mathrm{d}}$; $\mathrm{I}_{\mathrm{Nm}}=$ matriz identidade de dimensão $\mathrm{N}_{\mathrm{m}} ; \otimes=$ produto de Kronecker; $\mathrm{R}$ = matriz diagonal de variâncias residuais com elementos que dependem da estrutura empregada, ou seja, $R=\operatorname{diagonal}\left[\sigma_{j}^{2}\right]$. A covariância entre os efeitos genéticos aditivos direto e materno foi assumida como igual a zero.

As covariâncias entre os coeficientes de regressão aleatória genéticos aditivos direto e materno e ambiente permanente de animal e materno, conforme o modelo ajustado, foram estimadas pelo método da máxima verossimilhança restrita (REML), usando o programa DXMRR do software DFREML (Meyer, 1998).

Os diferentes modelos foram comparados pelo teste da razão de verossimilhança (LRT), critério de informação de
Akaike (AIC) e o critério de informação Bayesiano de Schwarz (BIC). Os critérios AIC e BIC foram obtidos por: $A I C=-2 \ln L+2 p$ e $B I C=-2 \ln L+p \ln (N-r)$ em que p se refere ao número de parâmetros do modelo; $\mathrm{N}$ = número total de observações; e $r$ = posto da matriz de incidência para os efeitos fixos. Menores valores de AIC e BIC e maiores para o Log L indicam modelos mais adequados. A estatística LRT foi obtida pela seguinte expressão: $L_{R T} T_{i j}=2 \log L_{i}-2 \log$ $L_{j}$, em que $\log L_{i}=$ máximo da função de verossimilhança para o modelo completo i, e $\log L_{j}=$ máximo da função de verossimilhança para o modelo reduzido $\mathrm{j}$. A estimativa LRT foi comparada com o valor do qui-quadrado tabelado, com d graus de liberdade e nível de significância de 1\%, em que d a diferença entre o número de parâmetros estimados pelos modelos completo e reduzido (modelos aninhados).

Avaliaram-se as alterações visuais ocorridas nos componentes de variância estimados e parâmetros genéticos resultantes ao longo da curva de crescimento, em função dos diferentes modelos para ajuste da variância residual, como também no ordenamento dos animais a partir dos valores genéticos preditos para peso ao nascimento, ao desmama e aos 196 dias de idade, por meio da estimativa do coeficiente de correlação de Spearman.

\section{Resultados e Discussão}

Comparando, inicialmente, a modelagem do resíduo por meio de classes, observaram-se aumentos no Log L, significativos $(\mathrm{P}<0,01)$ pelo teste da razão de verossimilhança (LRT), com o aumento do número de classes heterogêneas (Tabela 1). De acordo com todos os critérios utilizados para avaliar a qualidade de ajuste, o modelo considerando homogeneidade de variâncias residuais mostrou-se inadequado. Pelo LRT, quando se comparou o modelo homogêneo ao modelo CL2, a diferença entre ambos foi altamente significativa $(\mathrm{P}<0,01)$, indicando que CL2 proporcionou melhor modelagem da variância residual.

Mudanças consideráveis nos valores do Log L, AIC e BIC, como também significativas $(\mathrm{P}<0,01)$ pelo LRT, ocorreram até o modelo CL5 (Tabela 1). Ao observar o Log L e o AIC para os modelos CL5 até CL7, verificaram-se pequenas alterações nos seus valores ( $\mathrm{P}>0,01$ pelo LRT), sugerindo pouca melhoria de ajuste, embora o melhor valor de AIC tenha sido obtido com o modelo CL7, porém a diferença em relação a CL5 foi pequena. O menor valor para BIC foi obtido com o modelo CL5, sugerindo que as cinco classes foram suficientes para modelar a variância residual. O resultado apresentado pelo BIC é consistente, já que as maiores diferenças ocorreram entre modelos com menos de cinco classes de variâncias residuais. 
Vale ressaltar que outros modelos com maior número de classes foram comparados (resultados não apresentados). Entretanto, as diferenças nas estimativas de variância em relação às classes (Tabela 1), foram muito pequenas, indicando que a utilização de modelos com mais de cinco classes residuais não resultaria em grandes alterações na modelagem do resíduo.

Lewis \& Brotherstone (2002) verificaram que a variância residual com seis classes heterogêneas (sendo as classes formadas com intervalos de 25 dias) proporcionou melhor ajuste, quando comparado ao modelo com variância residual constante. Já Fischer et al. (2004) modelaram a variância residual com nove classes heterogêneas, sendo as classes definidas a cada cinquenta dias de idade.

Nos modelos em que se empregaram funções de variâncias residuais, utilizando-se polinômios ortogonais de Legendre de diferentes ordens, observaram-se melhores valores para o $\log \mathrm{L}(\mathrm{P}<0,01)$, AIC e BIC com o aumento da ordem da função. Entre as ordens avaliadas, a quinta (FL5) apresentou menor valor para todos os critérios, indicando melhor ajuste. Todavia, os critérios apresentaram dificuldade de convergência, onde a função com ordem seis não convergiu, mesmo quando se tentou reiniciar as análises com diversos valores iniciais para os parâmetros. Relatos de dificuldade de convergência com funções de variâncias empregando polinômios de Legendre foram mencionados por Lewis \& Brotherstone (2002), os quais comentaram que a convergência não foi alcançada quando esta modelagem

Tabela 1 - Número de parâmetros (NP), Logaritmo da função de máxima verossimilhança $(\log \mathrm{L})$, critério de informação de Akaike (AIC), critério de informação Bayesiano (BIC) e teste da razão de verossimilhança (LRT) estimados com os modelos estudados

\begin{tabular}{lccccc}
\hline Modelo & NP & Log L $^{1}$ & AIC $^{2}$ & BIC $^{2}$ & LRT \\
\hline CL1 & 25 & -964 & 3168 & 3129 & (CL2-CL1) 812, $2^{*}$ \\
CL2 & 26 & -558 & 2358 & 2326 & (CL3-CL2) 867, 2* \\
CL3 & 27 & -124 & 1492 & 1469 & (CL4-CL3) 155,8* \\
CL4 & 28 & -46 & 1339 & 1323 & (CL5-CL4) 88,0* \\
CL5 & 29 & $-2,2$ & 1253 & 1245 & (CL6-CL5) 0,3 ns \\
CL6 & 30 & $-1,9$ & 1254 & 1254 & (CL7-CL5) 4,4 ns \\
CL7 & 31 & 0 & 1252 & 1260 & - \\
FL2 & 26 & -157 & 316 & 284 & (FL3-FL2) $84^{*}$ \\
FL3 & 27 & -115 & 235 & 211 & (FL4-FL3) $8^{*}$ \\
FL4 & 28 & -111 & 227 & 212 & (FL5-FL4) $220^{*}$ \\
FL5 & 29 & 0 & 9 & 1 & - \\
FO2 & 26 & -162 & 316 & 284 & (FO3-FO2) $84^{*}$ \\
FO3 & 27 & -120 & 234 & 211 & (FO4-FO3) 62* \\
FO4 & 28 & -89 & 173 & 158 & (FO5-FO4) $124^{*}$ \\
FO5 & 29 & -27 & 51 & 43 & (FO6-FO5) $54^{*}$ \\
FO6 & 30 & 0 & 0 & 0 & - \\
\hline
\end{tabular}

* Significativo a $1 \%$ de probabilidade; ${ }^{\text {ns }}$ não-significativo; ${ }^{1}$ Valores expressos como desvio do melhor valor, dentro de cada estrutura; ${ }^{2}$ Valores expressos como desvio do melhor valor.

$\mathrm{CL}$ = classes de idade; $\mathrm{FL}=$ polinômio de Legendre; $\mathrm{FO}$ = polinômio ordinário. foi empregada para o resíduo, corroborando os achados de El Faro \& Albuquerque (2003).

Os modelos com funções de variância ajustadas por meio de polinômios ordinários apresentaram convergências mais rápidas no processo iterativo. Comparando-se as diferentes ordens, observaram-se aumentos nos valores do $\log \mathrm{L}(\mathrm{P}<0,01)$ e diminuição nos valores de AIC e BIC com o aumento da ordem de ajuste, sugerindo que o modelo de maior ordem proporcionou melhor ajuste.

Com base nos dados apresentados (Tabela 1) e como se pode observar (Figura 1), assumir homogeneidade de variância residual ao longo da curva crescimento pode criar distorções consideráveis na partição da variância total, uma vez que as diferenças nas variâncias residuais estimadas antes e depois dos 56 dias de idade, quando comparadas com as estimativas considerando heterogeneidade, foram grandes.

Dentre os modelos que assumiram heterogeneidade, o ajuste obtido com FO6, como indicado pelos valores dos critérios AIC e BIC, foi melhor que o ajuste com cinco classes de variâncias residuais. A função FL5 estimou variâncias residuais próximas às estimadas por FO6 até a desmama; após esse ponto, as variâncias aumentaram até os 168 dias e diminuíram até os 196 dias, estimando-se a menor variância dentre as estruturas comparadas nesta fase. Todavia, os valores do BIC para FL5 e FO6 (Tabela 1) sugerem que as variâncias praticamente não diferiram, apesar do maior número de parâmetros de FO6.

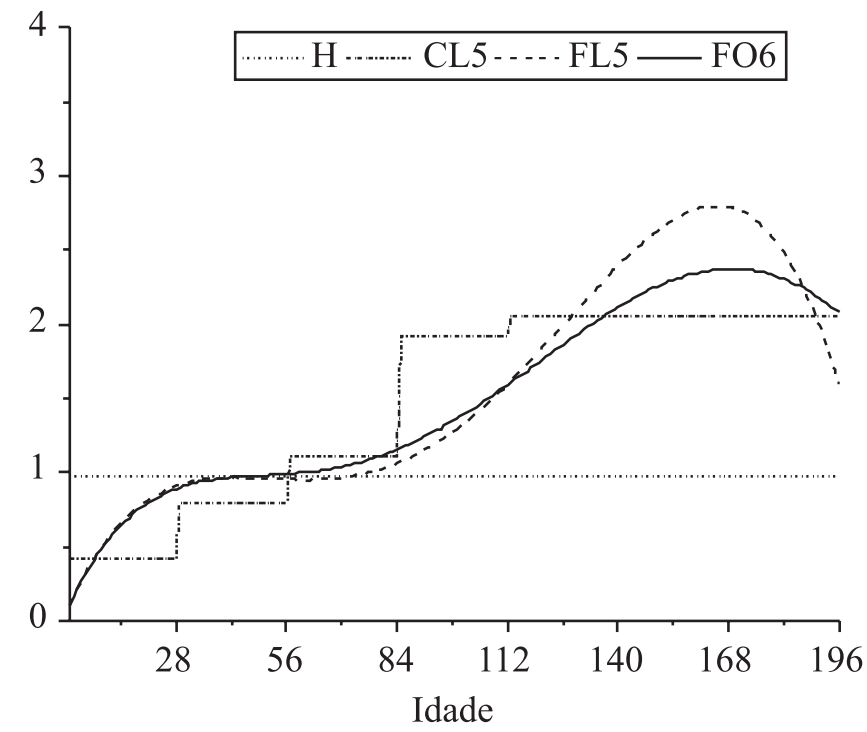

Figura 1 - Estimativas de variâncias residuais homogêneas (H) e heterogêneas, por meio de classes (CL5) e funções de variâncias (FL5 e FO6). 
As variâncias fenotípicas estimadas ao longo da curva de crescimento, quando se considerou a variância residual homogênea e heterogênea, diferiram no início e depois dos 84 dias até o final da curva. Como se observa (Figura 2), quando o resíduo foi assumido como homogêneo, a variância fenotípica foi ligeiramente maior no início e menor do meio para o final da curva que as estimadas com as duas funções. As duas funções com estruturas heterogêneas estimaram variâncias fenotípicas próximas depois dos 84 dias de idade, com tendência da FL5 estimar variâncias ligeiramente maiores no final da curva. Além disso, as duas funções com heterogeneidade estimaram variâncias fenotípicas ligeiramente superiores às estimadas em análises univariadas, principalmente depois dos 84 dias de idade, embora de comportamentos semelhantes.

As variâncias devido aos efeitos de animal, ou seja, genética aditiva direta e ambiente permanente de animal, foram mais influenciadas pelo ajuste da variância residual que as devido ao efeito materno (genética materna e ambiente permanente da mãe). Observa-se (Figura 3) que a variância genética aditiva direta estimada com homogeneidade de variância residual foi inferior, durante todo período de estudo, à estimada pelos modelos considerando heterogeneidade (FL5 e FO6). Observou-se, ainda, que a diferença entre as duas modelagens aumentou com a idade. Já com relação à variância devido ao ambiente permanente, o modelo considerando homogeneidade proporcionou estimativas superiores, a partir dos 56 dias de idade, às obtidas considerando heterogeneidade de variância residual. Da mesma forma como observado para o efeito genético aditivo direto, a diferença nas estimativas

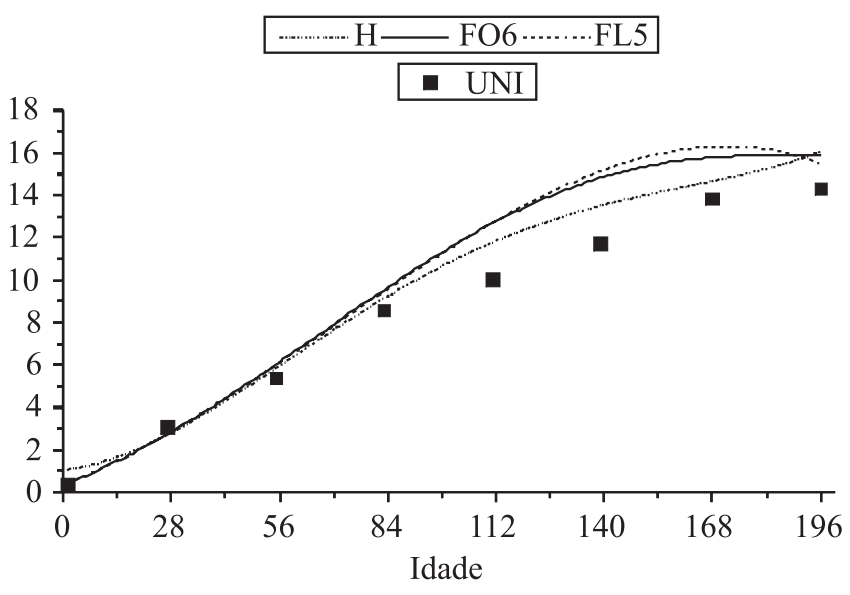

Figura 2 - Estimativas de variâncias fenotípicas em função da modelagem da variância residual, homogênea $(\mathrm{H})$ e heterogêneas (FL5 e FO6), e estimadas em análises univariadas (UNI). da variância de ambiente permanente de animal aumentou com a idade. Observa-se também na Figura 4 que os modelos FL5 e FO6 não diferiram com relação às estimativas das variâncias de animal.

As estimativas de herdabilidades diretas ao longo da curva de crescimento diferiram em função do ajuste da variância residual (Figura 4). As herdabilidades estimadas quando a variância residual foi assumida como heterogênea, não havendo diferença entre os modelos com FL5 e FO6, foram todas superiores às estimadas com o resíduo constante e com comportamentos diferentes no início da trajetória. Com o resíduo constante, as herdabilidades estimadas foram crescentes do nascimento (herdabilidade próxima de zero) aos 140 dias (com valor igual a 0,13), apresentando ligeiro decrescimento em seguida, chegando a 0,1 aos 196 dias de idade. Já ajustando as mudanças da variância residual com o tempo, a herdabilidade decresceu do nascimento $(0,19)$ até próximo aos 15 dias $(0,1)$, aumentou deste ponto até por volta dos 120 dias $(0,17)$, onde atingiu o valor máximo, e decresceu em seguida, alcançando herdabilidade de 0,15 aos 196 dias de idade. Oliveira et al. (2010) estimaram herdabilidade ao nascimento e aos 50 dias de idade de 0,24 e 0,12, respectivamente, em ovinos deslanados por meio de modelos de regressão aleatória. Herdabilidades próximas às estimadas para pesos ao nascimento têm sido reportadas em ovinos de corte empregando-se tradicionais modelos de análise (Snyman et al., 1995; Sarmento et al., 2006).

As herdabilidades maternas estimadas em função da modelagem da variância residual (homogênea e heterogênea) diferiram, principalmente, do nascimento aos 15 dias de idade. As estimativas de herdabilidade materna obtidas com os modelos considerando homogeneidade e heterogeneidade de variância residual divergiram nos primeiros 15 dias, com tendência das estimadas com resíduo constante serem maiores após esta idade (Figura 4). Da mesma forma que as variâncias maternas, os modelos que consideraram heterogeneidade não diferiram.

As herdabilidades direta e materna estimadas pelo modelo com homogeneidade saíram de praticamente zero, próximo ao nascimento, para 0,18 com o modelo assumindo heterogeneidade. A estimativa de herdabilidades maternas diferentes de zero ao nascimento é biologicamente coerente, pois a mãe exerce influência sobre a cria desde o período de gestação, ou seja, a contribuição do efeito materno não começa a partir do nascimento do animal, como sugerido pelas estimativas obtidas com modelo em que o resíduo permaneceu constante.

Além disso, observa-se (Figura 4) que as estimativas de herdabilidade materna obtidas com o modelo que 
considerou resíduo constante foram sempre maiores que as estimativas de herdabilidade direta, o que não era esperado. Quando a variância residual foi considerada heterogênea (ao se ajustar os modelos FL5 e FO6), as estimativas de herdabilidade materna, do terceiro aos 28 dias de idade, foram superiores à direta, sugerindo que, neste período, a mãe exerceu maior influência sobre a prole. A partir dos 28 dias, a herdabilidade materna cresceu até os 84 dias, a partir de onde as estimativas de herdabilidade direta passaram a ser maiores até o final da curva. Esse comportamento entre as trajetórias das herdabilidades direta e materna é biologicamente correto, pois, à medida que o cordeiro aumenta de idade, torna-se mais dependente do próprio potencial genético para crescer, conforme descrito por Snyman et al. (1995), Sousa et al. (1999) e Sarmento et al. (2006).
Após os 84 dias de idade, as estimativas de herdabilidade materna decresceram, como esperado. Todavia, a expectativa era de que a contribuição do efeito materno após os 112 dias de idade fosse menor. Vale ressaltar que esse resultado pode ter sido obtido devido à falta de um modelo com as ordens apropriadas para as funções contínuas que descreveram as variâncias associadas a cada efeito aleatório, o que pode acarretar problemas na partição da variância total. Além disso, com a proximidade do final da curva, o número de observações tende a diminuir o que, também, pode ocasionar problemas de partição nas variâncias.

O ambiente permanente de animal como proporção da variância fenotípica estimado com homogeneidade e heterogeneidade de variância residual diferiu, praticamente, durante todo o período estudado (Figura 4). Até os 28 dias de idade, o efeito do ambiente permanente de animal estimado

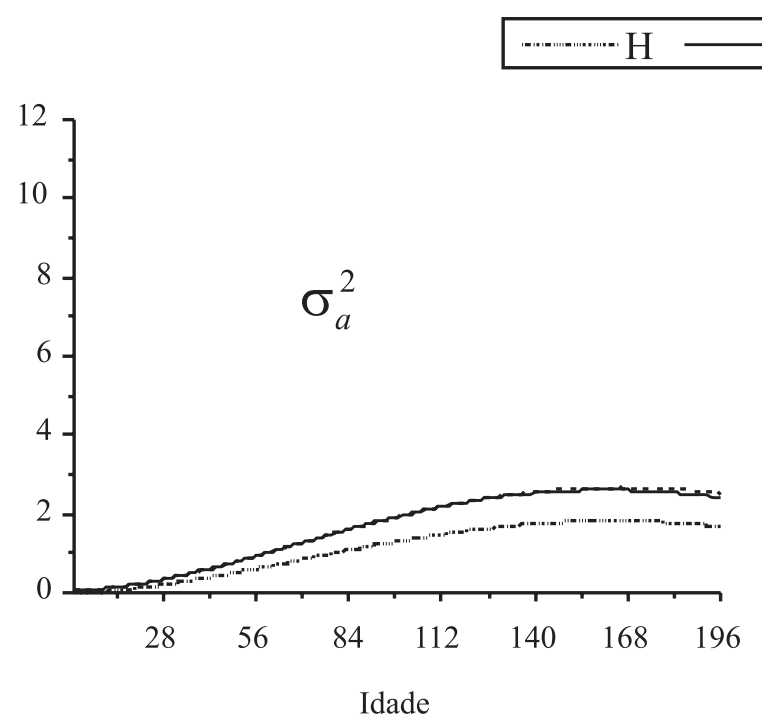

FO6 $\cdots \cdots \cdots$ FL5
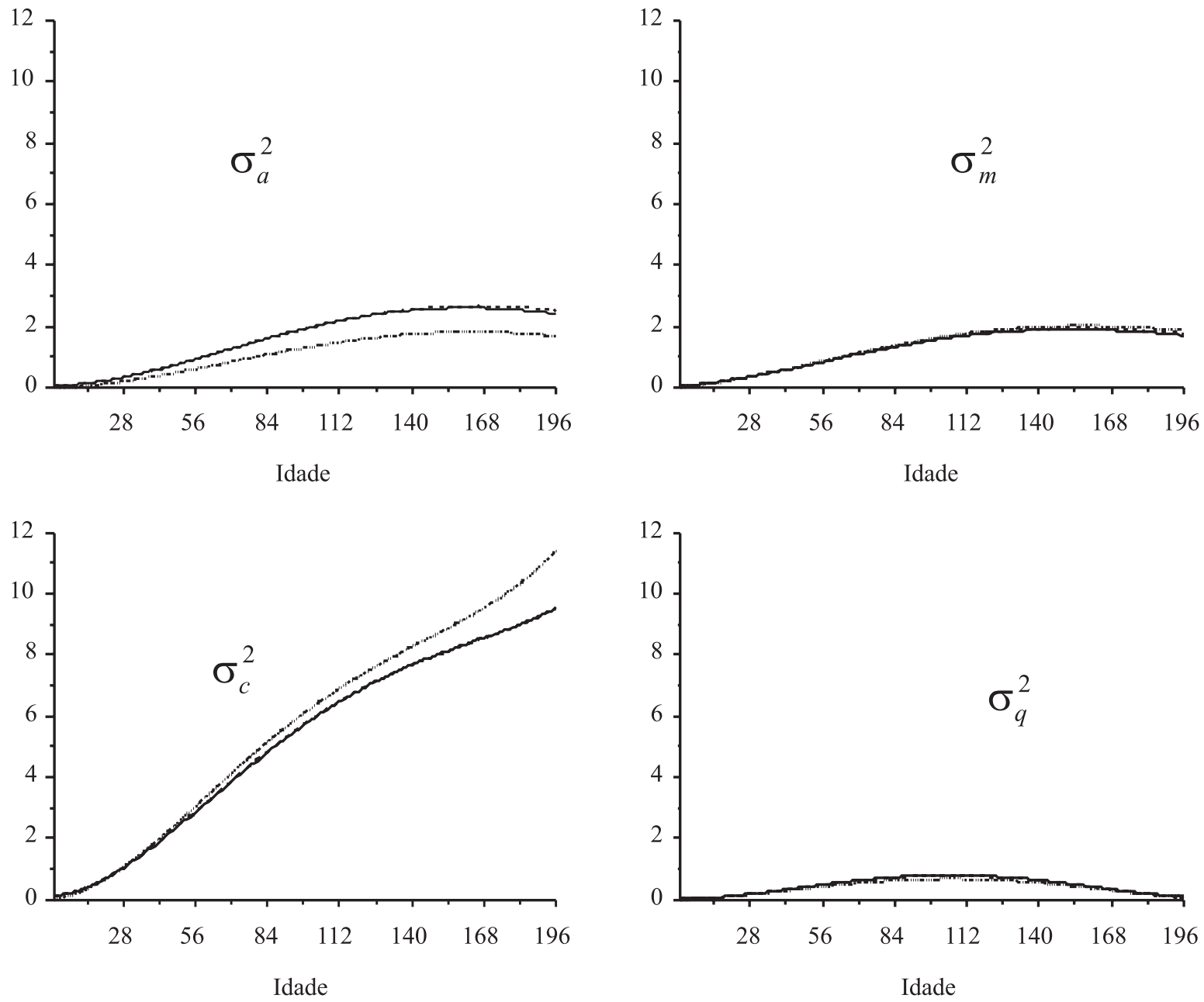

Figura 3 - Estimativas de componentes de variância genética direta $\left(\sigma_{a}^{2}\right)$, genética materna $\left(\sigma_{m}^{2}\right)$, ambiente permanente de animal $\left(\sigma_{c}^{2}\right)$ e ambiente permanente materno $\left(\sigma_{q}^{2}\right)$, em função da modelagem da variância residual, homogênea (H) e heterogênea (FL5 e FO6). 
pelo modelo com homogeneidade foi inferior, ao passo que após os 28 dias esse efeito passou a ser superior ao estimado pelo modelo, considerando a variância residual heterogênea. Assumindo que a partição da variância total foi feita corretamente com heterogeneidade (FL5 ou FO6), estes resultados sugerem que houve subestimação e superestimação, antes e depois dos 28 dias de idade, para este efeito.

Para o efeito de ambiente permanente da mãe, como proporção da variância fenotípica, houve uma pequena diferença nas estimativas obtidas com as duas modelagens da variância residual, onde as estimadas no início da curva pelo modelo com heterogeneidade foram ligeiramente maiores (Figura 4).

As estimativas de correlações genéticas diretas entre pesagens em idades do nascimento aos 196 dias (Figura 5) também foram afetadas pela mudança na modelagem das variâncias residuais. As maiores alterações foram verificadas entre as correlações de pesos no início da curva com os demais pesos, sendo que as estimadas quando se manteve a variância residual constante, foram todas próximas à unidade. As correlações estimadas por meio dos modelos FL5 e FO6 não diferiram, sendo maiores entre idades adjacentes.

As correlações genéticas maternas estimadas também foram alteradas com a modelagem da variância residual. Verificou-se tendência de suavização, ou seja, as correlações diminuíram gradativamente com o aumento de tempo entre as idades (Figura 5), quando o resíduo mudou em função do tempo. As estimativas obtidas com os modelos FL5 ou FO6 também não diferiram.

O mesmo comportamento verificado com as estimativas de correlações genéticas maternas foi obtido com as

\section{….. H FO6 -...... FL5}
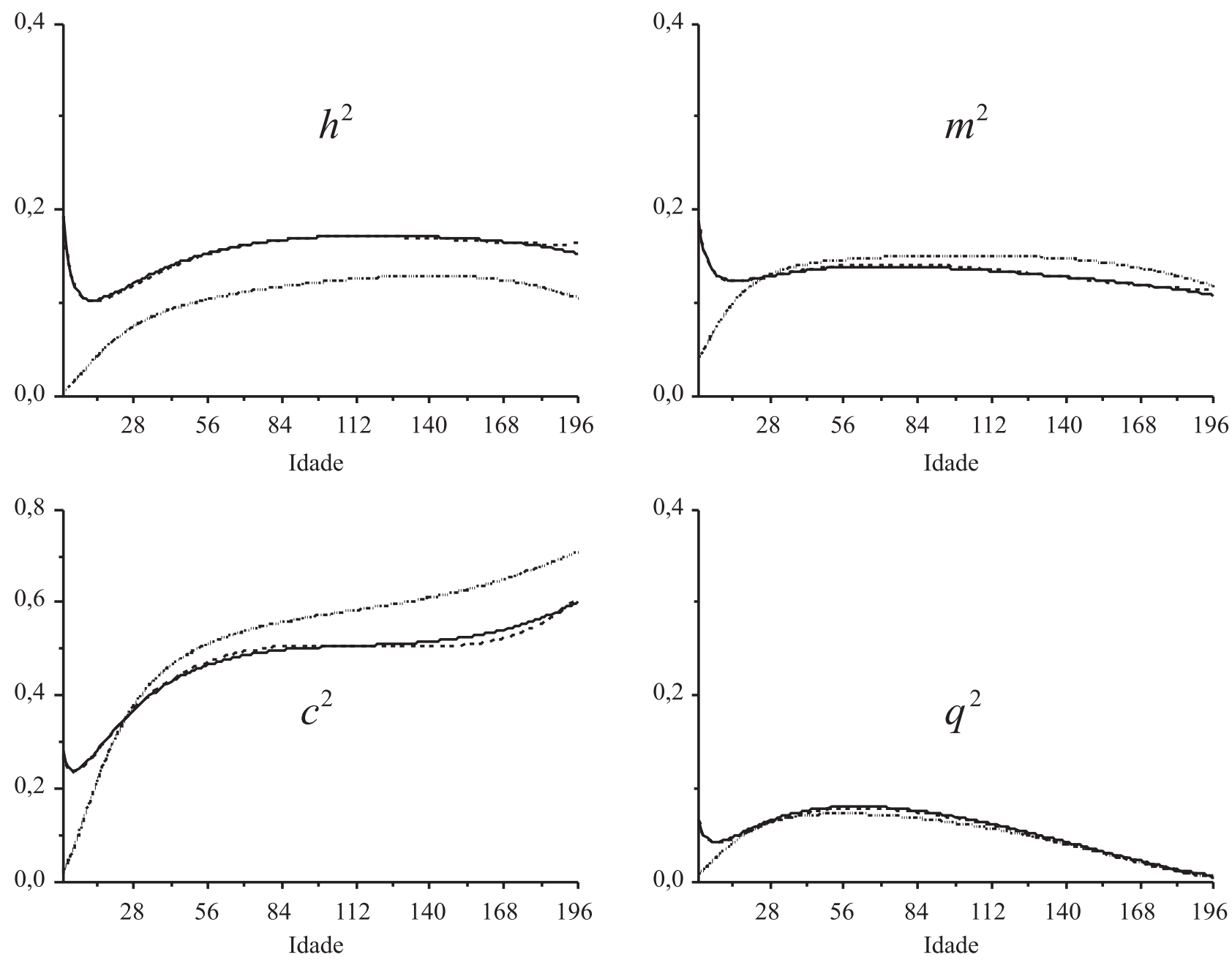

Figura 4 - Estimativas de herdabilidade direta $\left(\mathrm{h}^{2}\right)$ e materna $\left(\mathrm{m}^{2}\right)$ e componentes de variância para ambiente permanente de animal $\left(\mathrm{c}^{2}\right)$ e materno $\left(\mathrm{q}^{2}\right)$, como proporção da variância fenotípica, em função da modelagem da variância residual, como homogênea (H) e heterogênea (FL5 e FO6). 
estimativas de correlações de ambiente permanente de animal, porém de magnitudes diferentes (Figura 5). As correlações estimadas aumentaram à medida que as idades entre as pesagens se aproximavam.

A modelagem da variância residual também afetou os valores genéticos preditos, o que era esperado devido às alterações nas estimativas de variâncias e herdabilidades. As correlações de ordem entre os valores genéticos preditos sob homogeneidade e heterogeneidade do resíduo para peso ao nascimento foi de apenas 0,23 e 0,24, respectivamente, com os modelos FO6 e FL5; já para o peso aos 112 e aos 196 dias de idade, essas correlações foram altas, 0,97
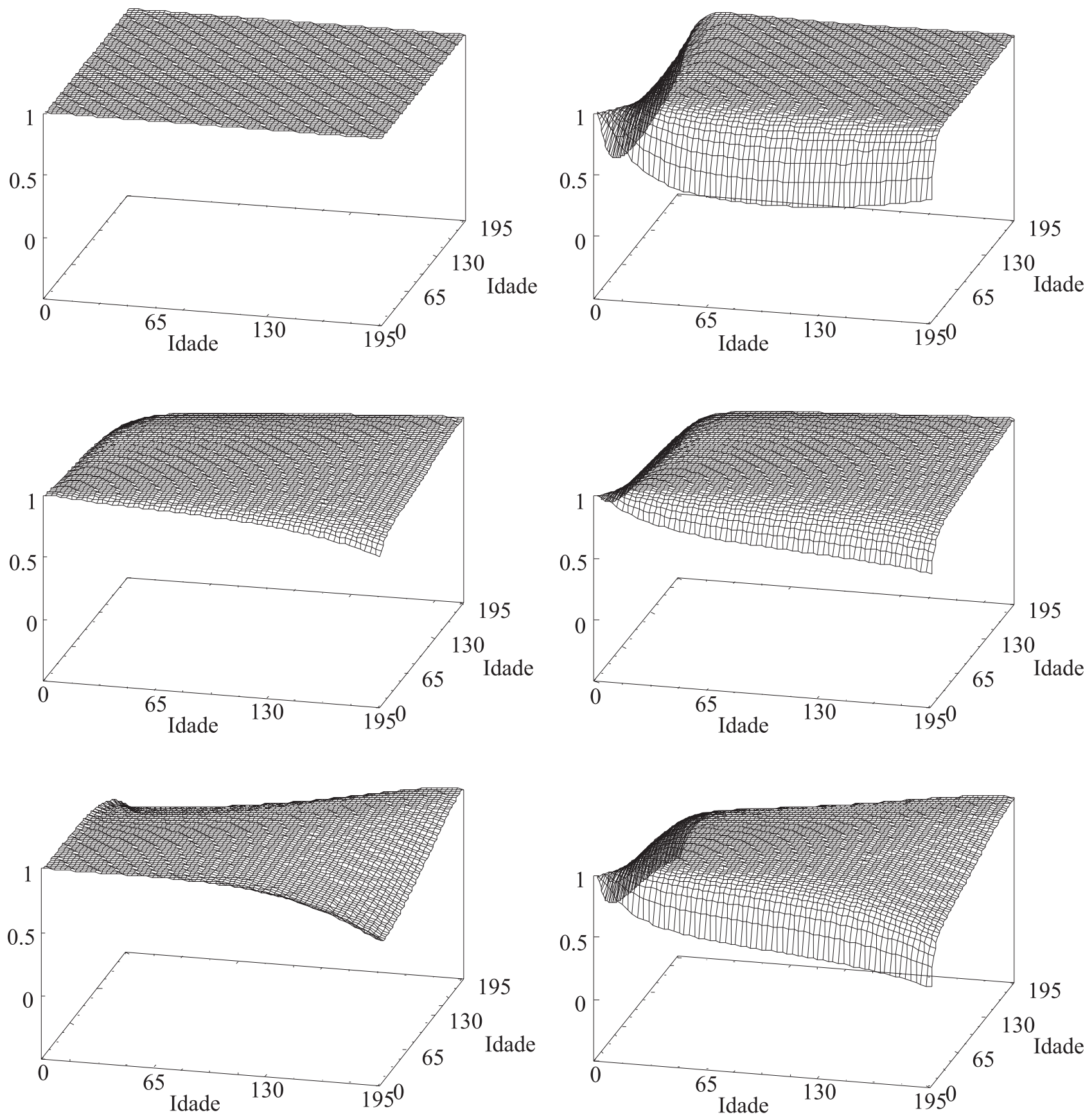

Figura 5 - Estimativas de correlações genética direta (acima), genética materna (no meio) e de ambiente permanente de animal (abaixo) estimadas assumindo homogeneidade (à esquerda) e heterogeneidade de variância residual (à direita), obtidas com o modelo FL5. 
e 0,93, com FO6 e FL5, na mesma ordem. As correlações de ordem entre os valores genéticos preditos para peso ao nascimento, 112 e 196 dias de idade, entre os modelos FO6 e FL5, foram todas próximas da unidade. Ao analisar os valores das correlações, constata-se que as alterações nas classificações foram em maior proporção para os pesos do início e final da curva, uma vez que nessas extremidades a variância residual foi mais afetada pela modelagem empregada.

Alterações consideráveis na magnitude dos valores genéticos preditos também foram verificadas em função da modelagem do resíduo. Observa-se (Tabela 2) que os valores genéticos preditos quando se assumiu homogeneidade foram todos subestimados em relação aos preditos quando a variância residual foi ajustada com polinômio ordinário de ordem seis (FO6). Já em relação aos valores genéticos preditos com os modelos que assumiram variância residual heterogênea, FL5 em relação a FO6, as diferenças foram muito pequenas. Verificou-se, ainda, que as maiores diferenças entre o ajuste homogêneo e heterogêneo foram obtidas com o peso ao nascimento, com diferenças maiores que $94 \%$, possivelmente pelo fato de a modelagem da variância residual ter afetado as estimativas da variância genética aditiva em maior proporção nesta fase de crescimento. Os valores genéticos preditos para os pesos aos 112 e 196 dias apresentaram diferenças menores, todavia elas variaram de 27 a $31 \%$ e de 26 a 41\%, respectivamente, para os referidos pesos.

Tabela 2 - Valores genéticos preditos para os cinco melhores animais para pesos ao nascimento, aos 112 e 196 dias de idade, considerando homogeneidade e heterogeneidade de variância residual

\begin{tabular}{|c|c|c|c|c|}
\hline \multirow[t]{2}{*}{ Idade } & \multirow[t]{2}{*}{ Animal } & \multicolumn{3}{|c|}{ Valor genético predito (kg) } \\
\hline & & Homogeneidade $^{1}$ & $(\mathrm{FO6})$ & $\mathrm{FL}^{2}$ \\
\hline \multirow[t]{5}{*}{ Nascimento } & 5222 & $0,068(-87,1)$ & 0,526 & $0,527 \quad(0,19)$ \\
\hline & 59 & $0,055(-89,0)$ & 0,500 & $0,500(0)$ \\
\hline & 27 & $0,063(-87,2)$ & 0,492 & $0,489 \quad(-0,61)$ \\
\hline & 5383 & $0,028(-94,2)$ & 0,485 & $0,491 \quad(1,23)$ \\
\hline & 2123 & $0,031 \quad(-93,4)$ & 0,470 & $0,474 \quad(0,85)$ \\
\hline \multirow[t]{5}{*}{112 dias } & 2096 & $1,773(-31,6)$ & 2,591 & $2,580 \quad(-0,42)$ \\
\hline & 5407 & $1,754(-29,5)$ & 2,487 & $2,454 \quad(-1,32)$ \\
\hline & 18 & $1,654(-31,2)$ & 2,405 & $2,394 \quad(-0,45)$ \\
\hline & 3608 & $1,700(-28,6)$ & 2,380 & $2,360 \quad(-0,84)$ \\
\hline & 5350 & $1,680(-27,7)$ & 2,325 & $2,333(0,34)$ \\
\hline \multirow[t]{5}{*}{196 dias } & 2096 & $1,890(-29,5)$ & 2,679 & $2,726(1,75)$ \\
\hline & 5407 & $1,868(-29,8)$ & 2,660 & $2,685(0,93)$ \\
\hline & 1947 & $1,447 \quad(-41,7)$ & 2,480 & $2,567 \quad(3,50)$ \\
\hline & 2061 & $1,718(-29,1)$ & 2,423 & $2,464 \quad(1,69)$ \\
\hline & 18 & $1,762(-26,6)$ & 2,401 & $2,440 \quad(1,62)$ \\
\hline
\end{tabular}

1 Valores entre parênteses expressam os desvios, em porcentagem, dos valores genéticos preditos, considerando homogeneidade em relação aos preditos com FO6.

2 Valores entre parênteses expressam os desvios, em porcentagem, dos valores genéticos preditos com FL5 em relação aos preditos com FO6.

\section{Conclusões}

As estimativas de variâncias, herdabilidades, correlações e os valores genéticos preditos são afetados pela modelagem da variância residual. Portanto, é necessária a utilização de heterogeneidade de variâncias residuais para modelar as variâncias associadas à curva de crescimento dos ovinos Santa Inês em estudo. A melhor modelagem da variância residual é a que considerou uma função de variância com polinômios ordinários de ordem 6. Todavia, a função de variância que empregou um polinômio de Legendre de ordem 5 acomodou as mudanças nas variâncias residuais com a idade. A modelagem da variância residual por meio de classes é inferior ao ajuste proporcionado pelas funções de variâncias com polinômios ordinários e de Legendre.

\section{Referências}

ALBUQUERQUE, L.G.; MEYER, K. Estimates of covariance functions for growth from birth to 630 days of age in Nelore cattle. Journal of Animal Science, v.79, p.2776-2789, 2001.

EL FARO, L.; ALBUQUERQUE, L.G. Utilização de modelos de regressão aleatória para produção de leite no dia do controle, com diferentes estruturas de variâncias residuais. Revista Brasileira de Zootecnia, v.32, n.5, p.1104-1113, 2003.

FISCHER, T.M.; VAN DER WERF, J.H.J.; BANKS, R.G. et al. Description of lamb growth using random regression on field data. Livestock Production Science, v.89, p.175-185, 2004.

KIRKPATRICK, M.; LOFSVOLD, D.; BULMER, M. Analysis of the inheritance, selection and evolution of growth trajectories. Genetics, v.124, p.979-993, 1990.

LEWIS, R.M.; BROTHERSTONE, S. A genetic evaluation of growth in sheep using random regression techniques. Animal Science, v.74, p.63-70, 2002.

MEYER, K. "DXMRR" - A program to estimate covariance functions for longitudinal data by REML. In: WORLD CONGRESS OF GENETICS APPLIED TO LIVESTOCK PRODUCTION, 6., 1998, Armidale. Proceedings... Armidale: University of New England, 1998. (CD-ROM).

MEYER, K. Estimates of direct and maternal covariance functions for growth of Australian beef calves from birth to weaning. Genetics Selection Evolution, v.33, p.487-514, 2001.

MEYER, K. Estimates of genetic and phenotypic covariance functions for postweaning growth and mature weight of beef cows. Journal Animal Breeding Genetic, v.116, p.181-203, 1999.

MEYER, K. Estimates of genetic covariance functions for growth of Angus cattle. Journal Animal Breeding Genetic, v.122, p.73-85, 2005.

MEYER, K. Random regression to model phenotypic variation in monthly weights of Australian beef cows. Livestock Production Science, v.65, p.19-38, 2000.

SARMENTO, J.L.R.; TORRES, R.A.; SOUSA, W.H. et al. Estimação de parâmetros genéticos para características de crescimento de ovinos Santa Inês utilizando modelos uni e multicaracterísticas. Arquivo Brasileiro de Medicina Veterinária e Zootecnia, v.58, n.4, p.581-589, 2006

SARMENTO, J.L.R.; TORRES, R.A.; SOUSA, W.H. et al. Modelagem da curva média de crescimento de ovinos Santa Inês em modelos de regressão aleatória. Revista Brasileira de Zootecnia, v.39, 2010 (prelo). 
OLIVEIRA, K.A.; LÔBO, R.N.B.; FACÓ, O. Genetic evaluation of partial growth trajectory of Santa Inês breed using random regression models. Revista Brasileira de Zootecnia, v.39, n.5, p.1029-1036, 2010.

SNYMAN, M.A; ERASMUS, G.J.; VAN WYK, J.B. et al. Direct and maternal (co)variance components and heritability estimates for body weight different ages and fleece traits in
Afrino sheep. Livestock Production Science, v.44, p.229-235, 1995.

SOUSA, W.H.; PEREIRA, C.S.; BERGMANN, J.A.G. et al. Estimativas de componentes de (co)variância e herdabilidade direta e materna de pesos corporais em ovinos da raça Santa Inês. Revista Brasileira de Zootecnia, v.28, n.6, p.1252-1262, 1999. 